\title{
DENSE MOTION ESTIMATION BETWEEN DISTANT FRAMES: COMBINATORIAL MULTI-STEP INTEGRATION AND STATISTICAL SELECTION
}

\author{
Pierre-Henri Conze $e^{\star \dagger} \quad$ Tomás Crivelli ${ }^{\star} \quad$ Philippe Robert $^{\star} \quad$ Luce Morin $^{\dagger}$ \\ ${ }^{\star}$ Technicolor \\ ${ }^{\dagger}$ INSA Rennes, IETR/UMR 6164, UEB
}

\begin{abstract}
Accurate estimation of dense point correspondences between two distant frames of a video sequence is a challenging task. To address this problem, we present a combinatorial multistep integration procedure which allows one to obtain a large set of candidate motion fields between the two distant frames by considering multiple motion paths across the video sequence. Given this large candidate set, we propose to perform the optimal motion vector selection by combining a global optimization stage with a new statistical processing. Instead of considering a selection only based on intrinsic motion field quality and spatial regularization, the statistical processing exploits the spatial distribution of candidates and introduces an intra-candidate quality based on forward-backward consistency. Experiments evaluate the effectiveness of our method for distant motion estimation in the context of video editing.
\end{abstract}

Index Terms - motion estimation, statistical analysis, dense point matching, distant frames

\section{INTRODUCTION}

Despite rapid and significant progress since early formulations [1, 2], optical flow estimation still remains an open issue with crucial implications in computer vision. State-of-the-art methods $[3,4,5,6,7,8,9]$ have shown to be highly accurate for estimating dense motion fields between consecutive frames of a video sequence. However, they show limitations when applied to distant frames. The classical optical flow assumptions are not verified in this case, especially for difficult situations such as illumination changes, large motion, temporal occlusions, zooming, non-rigid deformations, low color contrast and transparency.

Direct matching between distant frames can be thus sensitive to ambiguous correspondences. An alternative consists in computing the long-range displacement through concatenation of elementary optical flow fields. This can be done by temporal integration, similarly to dense point tracking algorithms [7]. However, even small errors in the input fields can lead to large drifts in the final motion field.

A first step towards accurate long-range dense correspondences is to combine numerous estimations from direct matching and temporal integration. Following a similar approach to that presented in [10], one can select for each pixel the optimal motion vector among a set of candidate motion fields based on intrinsic motion field quality and spatial regularization. A more sophisticated processing, described in $[11,12]$, consists in sequentially merging a set of concatenated multi-step motion fields at intermediate frames up to the target frame. However, in either case, the optimal motion vector selection strongly depends on the same optical flow assumptions that frequently fail between distant frames. This issue could be partially compensated by complexifying the matching criteria ad-infinitum, but an uncertainty component is always present. This argues in favor of a statistical processing which takes into account the random nature of these perturbations among a large set of dense motion fields.

In this direction, we propose two main contributions to address the dense matching problem between distant frames. Firstly, we present a combinatorial multi-step integration method which allows one to get a large set of motion fields between two distant frames by considering multiple motion paths across the sequence. Secondly, once this motion candidate construction is performed, we apply a new approach to select the optimal motion field based on statistics and spatial regularization. Results for motion estimation between distant frames in the context of video editing are presented.

\section{MOTION CANDIDATE CONSTRUCTION}

Let us consider a sequence of $N+1$ RGB images $\left\{I_{n}\right\}_{n \in \llbracket 0, \ldots, N \rrbracket}$ and let $I_{a}$ and $I_{b}$ be two distant frames of this sequence with $0 \leq a<b \leq N$. In this paper, we focus on the frame pair $\left\{I_{a}, I_{b}\right\}$ and our goal is to accurately estimate a dense motion field between these two frames. In this section, we aim at generating multiple motion maps between $I_{a}$ and $I_{b}$.

\subsection{Direct matching with multiple optical flow estimators}

A first approach for building multiple motion candidates consists in considering a direct motion estimation using different optical flow methods [10]. Even if the considered estimators may fail in some regions, the idea is to pool the strengths of each one. Furthermore, the same estimator can be used several times by modifying its parameter settings. In addition, 


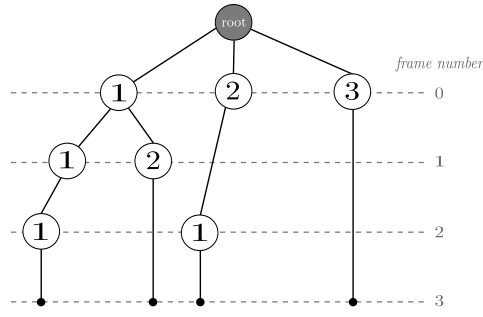

Fig. 1: Generation of step sequences. Going from the root node to leaf nodes of this tree structure gives $\boldsymbol{\Gamma}_{a, b}$, the set of possible step sequences from $I_{a}$ to $I_{b}$.

we can derive from each version a parametric motion field for which motion is constrained by a global transformation [13]. Direct matching is more ambiguous as the distance between $I_{a}$ and $I_{b}$ increases. Due to a large motion range, the motion of periodic color patterns or uniform areas may not be correctly estimated. This supports a motion field construction stage using concatenation of various optical flow fields.

\subsection{Combinatorial multi-step integration}

Let us describe the concept of motion path as an alternative to direct matching for obtaining a displacement map between $I_{a}$ and $I_{b}$. A motion path is obtained through concatenation of elementary optical flow fields across the video sequence. It links each pixel $\boldsymbol{x}_{a}$ of $I_{a}$ to a corresponding position in $I_{b}$. Elementary optical flow fields can be computed between consecutive frames or with different frame steps $[11,12]$, i.e. with larger inter-frame distances. Let $S_{n}=\left\{s_{1}, s_{2}, \ldots, s_{Q_{n}}\right\} \subset\{1, \ldots, N-n\}$ be the set of $Q_{n}$ possible steps at instant $n$. This means that the set of optical flow fields $\left\{\boldsymbol{v}_{n, n+s_{1}}, \boldsymbol{v}_{n, n+s_{2}}, \ldots, \boldsymbol{v}_{n, n+s_{Q_{n}}}\right\}$ is available.

Our objective is to produce a large set of motion maps between $I_{a}$ and $I_{b}$ as to form a significative set of samples upon which a statistical processing would be meaningful and advantageous. Given this objective, we propose to initially generate all the possible step sequences (i.e. combinations of steps) in order to join $I_{b}$ from $I_{a}$. Each step sequence defines a motion path. Let $\boldsymbol{\Gamma}_{a, b}=\left\{\gamma_{0}, \gamma_{1}, \ldots, \gamma_{K-1}\right\}$ be the set of $K$ possible step sequences $\gamma_{i}$ between $I_{a}$ and $I_{b}$. $\Gamma_{a, b}$ is computed by building a tree structure (Fig. 1) where each node corresponds to a motion field assigned to a given frame for a given step value (node value). In pratice, the construction of the tree is done recursively starting from $I_{a}$ : we create for each node as many children as the number of steps available at the current instant. A child node is not generated when $I_{b}$ has already been reached (therefore, the current node is considered as a leaf) or if $I_{b}$ is passed given the considered step. Finally, once the tree has been built, going from the root node to leaf nodes gives $\boldsymbol{\Gamma}_{a, b}$, the set of step sequences. For illustration, the tree in Fig. 1 indicates the four step sequences that can be generated going from $I_{0}$ to $I_{3}$ with steps 1,2 and 3: $\boldsymbol{\Gamma}_{0,3}=\{\{1,1,1\},\{1,2\},\{2,1\},\{3\}\}$.

Once all the possible step sequences $\gamma_{i} \forall i \in \llbracket 0, \ldots, K-$ $1 \rrbracket$ between $I_{a}$ and $I_{b}$ have been generated, the corresponding

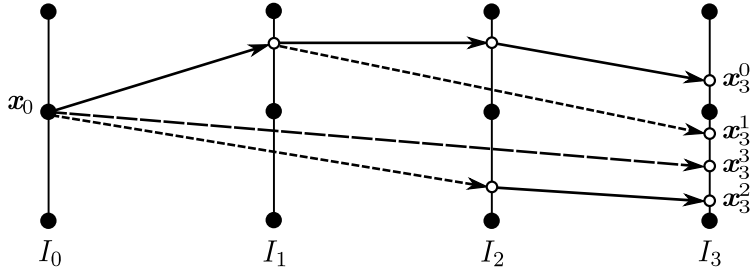

Fig. 2: Generation of multi-step motion paths. For each pixel $\boldsymbol{x}_{0}$ of $I_{0}$, this gives a set of candidate positions in $I_{3}$.

motion paths can be constructed through 1st-order Euler integration. Starting from each pixel $\boldsymbol{x}_{a}$ of $I_{a}$ and for each step sequence, this integration performs the accumulation of optical flow fields following the steps which form the current step sequence. Thus, with steps 1, 2 and 3, Fig.2 illustrates the construction of the four possible motion paths (one for each step sequence of $\boldsymbol{\Gamma}_{0,3}$ ) between $I_{0}$ and $I_{3}$. Let $f_{j}^{i}=a+\sum_{k=0}^{j} s_{k}^{i}$ be the current frame number during the construction of motion path $i$ from $I_{a}$ where $j$ is the step index within the step sequence $\gamma_{i}$. For each $\gamma_{i} \in \Gamma_{a, b}$ and for each step $s_{j}^{i} \in \gamma_{i}$, we start from $x_{a}$ in order to iteratively compute:

$$
\boldsymbol{x}_{f_{j}^{i}}=\boldsymbol{x}_{f_{j-1}^{i}}+\boldsymbol{v}_{f_{j-1}^{i}, f_{j}^{i}}\left(\boldsymbol{x}_{f_{j-1}^{i}}\right)
$$

Once all the steps $s_{j}^{i} \in \gamma_{i}$ have been run through, we obtain $\boldsymbol{x}_{b}^{i}$, the corresponding position in $I_{b}$ of $\boldsymbol{x}_{a}$ of $I_{a}$ obtained with step sequence $\gamma_{i}$. By considering all the step sequences, we finally get a large set of candidate positions in $I_{b}$ and this for each pixel $\boldsymbol{x}_{a}$ of $I_{a}$. Note that the occlusion maps attached to input motion fields are used to possibly stop the motion path construction. Considering an intermediate point $\boldsymbol{x}_{f_{j}^{i}}$ during the construction, a step can be added only if the closest pixel to $\boldsymbol{x}_{f_{j}^{i}}$ is considered as un-occluded for this step. Otherwise, the motion path is removed. In the following, the large set of candidate positions in $I_{b}$ is defined as $T_{a, b}\left(\boldsymbol{x}_{a}\right)=\left\{\boldsymbol{x}_{b}^{i}\right\}$ $\forall i \in \llbracket 0, \ldots, K_{\boldsymbol{x}_{a}}-1 \rrbracket$ where $K_{\boldsymbol{x}_{a}}$ is the cardinal of $T_{a, b}\left(\boldsymbol{x}_{a}\right)$.

Up to now, we have considered an exhaustive generation of step sequences for clarity. However, for very distant frames and for a large set of steps, it is not possible to consider all possible step sequences (computational and memory issues). For instance, for a distance of 30 frames and with steps 1, 2, 5 and 10, the number of possible motion paths is 5877241 . Therefore, the procedure described above is performed on a reasonable number of step sequences and not for all as previouly assumed. Firstly, we limit the number of elementary vectors composing the motion paths by providing a maximum number of concatenations $N_{c}$. Indeed, the concatenation of numerous vectors may lead to an important drift. Secondly, we randomly select $N_{s}$ motion paths among the remaining motion paths ( $N_{s}$ determined by storage capacity). This selection is guided by the fact that the candidate vectors should not be highly correlated. The frequency of appearance of a given step at a given frame must be uniform among all the possible steps arising from this frame in order to avoid a systematic bias towards the more populated branches of the tree. 


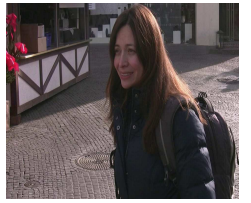

(a) $I_{25}$

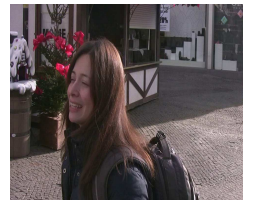

(b) $I_{40}$

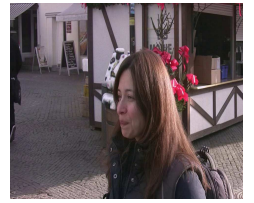

(c) $I_{55}$

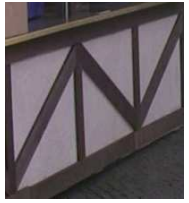

(d) $I_{46}$

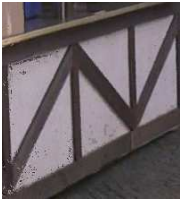

(e) $S P$

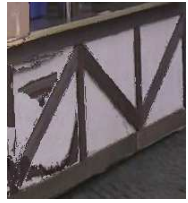

(f) $G O$

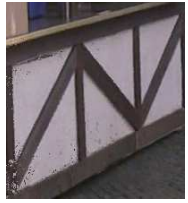

(g) $S P+G O$

Fig. 3: Source frames of the MPI S1 sequence [14] and reconstruction of the kiosk of $I_{46}$ from $I_{25}$ with: 1) the statistical processing $(S P), 2)$ the global optimization $(G O)$ method solved by fusion moves [15], 3) both combined $(S P+G O)$.

\subsection{Joint forward and backward processing}

Motion estimation can be enhanced by considering both forward and backward motion fields. Similarly to the forward direction, the set of backward motion fields from each pixel $\boldsymbol{x}_{b}$ of $I_{b}$ to $I_{a}$ can be computed by considering multi-step backward motion paths. These backward motion fields can be inversed into forward motion fields in order to enrich $T_{a, b}\left(\boldsymbol{x}_{a}\right)$. Thus, backward motion vectors from pixels of $I_{b}$ are projected into $I_{a}$. For each one, we identify the nearest pixel of the arrival position. The corresponding vector from $I_{b}$ to $I_{a}$ is reversed and started from the previously identified nearest pixel which gives a new candidate for $T_{a, b}\left(\boldsymbol{x}_{a}\right)$. Candidates of $T_{a, b}\left(\boldsymbol{x}_{a}\right)$ which have been obtained through this procedure are defined as reverse. Otherwise, we call them direct.

\section{MOTION VECTOR SELECTION ON LARGE SETS}

\subsection{Statistical processing for motion vector selection}

Given $T_{a, b}\left(\boldsymbol{x}_{a}\right)=\left\{\boldsymbol{x}_{b}^{i}\right\}_{i \in \llbracket 0, \ldots, K_{x_{a}}-1 \rrbracket}$, the set of candidate positions in $I_{b}$ obtained for each pixel $\boldsymbol{x}_{a}$ of $I_{a}$ from the motion field construction stage described above, the objective is to select the optimal candidate position $\boldsymbol{x}_{b}^{*} \in T_{a, b}\left(\boldsymbol{x}_{a}\right)$ by exploiting the statistical information on the point distribution and the quality of each candidate. The idea is to assume a Gaussian model for the distribution of $T_{a, b}\left(\boldsymbol{x}_{a}\right)$ and try to find its central value, $\boldsymbol{x}_{b}^{*}$. Using the maximum likelihood estimator (MLE) and imposing the selection among elements of $T_{a, b}\left(\boldsymbol{x}_{a}\right)$, the choice of the optimal candidate position $\boldsymbol{x}_{b}^{*}$ is defined by:

$$
\boldsymbol{x}_{b}^{*}=\arg \min _{\boldsymbol{x}_{b}^{i}} \sum_{\substack{j=0 \\ j \neq i}}^{K_{x_{a}}-1}\left\|\boldsymbol{x}_{b}^{j}-\boldsymbol{x}_{b}^{i}\right\|_{2}^{2}
$$

The assumption of Gaussianity can be largely perturbed by outliers. Consequently, a robust estimation of the distribution central value is necessary:

$$
\boldsymbol{x}_{b}^{*}=\arg \min _{\boldsymbol{x}_{b}^{i}} \operatorname{med}_{j \neq i}\left\|\boldsymbol{x}_{b}^{j}-\boldsymbol{x}_{b}^{i}\right\|_{2}^{2}
$$

Finally, each candidate position $\boldsymbol{x}_{b}^{i}$ receives a corresponding quality score $Q\left(\boldsymbol{x}_{b}^{i}\right)$ computed using the inconsistency values $\operatorname{Inc}\left(\boldsymbol{x}_{b}^{i}\right)$. Inc $\left(\boldsymbol{x}_{b}^{i}\right)$ corresponds to the Euclidean distance to the nearest reverse (resp. direct) candidate among the distribution if $\boldsymbol{x}_{b}^{i}$ is direct (resp. reverse). We aim at assigning high quality to candidates for which the corresponding motion field between $I_{a}$ and $I_{b}$ is consistent with a motion

\begin{tabular}{|l|c|c|c|c|c|c|}
\hline Frame pairs & $\{25,45\}$ & $\{25,46\}$ & $\{25,47\}$ & $\{25,48\}$ & $\{25,49\}$ & $\{25,50\}$ \\
\hline$S P$ & 12.72 & 15.27 & 21.7 & 25.33 & $\mathbf{2 4 . 4 8}$ & 24.7 \\
\hline$G O$ & 11.19 & 14 & 11.14 & 13.7 & 21.7 & 22.22 \\
\hline SP+GO & $\mathbf{1 2 . 8 4}$ & $\mathbf{1 6 . 1 1}$ & $\mathbf{2 4 . 7 5}$ & $\mathbf{2 5 . 5 5}$ & 24 & $\mathbf{2 4 . 7 9}$ \\
\hline
\end{tabular}

Table 1: Comparison through registration and PSNR assessment between: 1$)$ the statistical processing $(S P), 2)$ the global optimization $(G O), 3) S P+G O$. PSNR scores are computed on the kiosk of MPI S1 (Fig. 3). Low PSNR for first pairs are due to the foreground object which degrades the estimation.

field between $I_{b}$ and $I_{a}$. Quality scores $Q\left(\boldsymbol{x}_{b}^{i}\right)$ are computed as follows: the maximum and minimum values of $\operatorname{Inc}\left(\boldsymbol{x}_{b}^{i}\right)$ among all candidates are mapped from 0 to a predefined integer $Q_{\max }$. Intermediate inconsistency values are mapped to the line defined by these two values and the result is rounded to the nearest integer: $Q\left(\boldsymbol{x}_{b}^{i}\right) \in \llbracket 0, \ldots, Q_{\max } \rrbracket$. The higher $Q\left(\boldsymbol{x}_{b}^{i}\right)$, the smaller $\operatorname{Inc}\left(\boldsymbol{x}_{b}^{i}\right)$. We aim at promoting candidates in the neighborhood of high quality candidates. In practice, $Q\left(\boldsymbol{x}_{b}^{i}\right)$ is used as a voting mechanism [16]: while computing the medians in Equation (3), each sample $\boldsymbol{x}_{b}^{j}$ is considered $Q\left(\boldsymbol{x}_{b}^{j}\right)$ times to set the occurrence of elements $\left\|\boldsymbol{x}_{b}^{j}-\boldsymbol{x}_{b}^{i}\right\|_{2}^{2}$ which enforces the forward-backward motion consistency.

The statistical processing being applied for each pixel independently, we describe in what follows a global optimization method which includes regularization.

\subsection{Global optimization for motion vector selection}

We perform a global optimization stage that fuses for each pixel motion candidates into a single optimal motion field, following the approach of [10]. We introduce $L=\left\{l_{\boldsymbol{x}_{a}}\right\}$ as a labeling of pixels $\boldsymbol{x}_{a}$ of $I_{a}$ where each label indicates one of the candidates of $T_{a, b}\left(\boldsymbol{x}_{a}\right)$. Let $d_{a, b}^{l_{x_{a}}}$ be the corresponding motion vectors of candidates of $T_{a, b}\left(\boldsymbol{x}_{a}\right)$. We define the following energy and minimize it with fusion moves $[10,15]$.

$$
\begin{array}{r}
E_{a, b}(L)=\sum_{\boldsymbol{x}_{a}} \rho_{d}\left(C\left(\boldsymbol{x}_{a}, d_{a, b}^{l_{x_{a}}}\left(\boldsymbol{x}_{a}\right)\right)+\operatorname{Inc}\left(\boldsymbol{x}_{a}+d_{a, b}^{l_{x_{a}}}\left(\boldsymbol{x}_{a}\right)\right)\right) \\
+\sum_{<\boldsymbol{x}_{a}, \boldsymbol{y}_{a}>} \alpha_{\boldsymbol{x}_{a}, \boldsymbol{y}_{a}} \cdot \rho_{r}\left(\left\|d_{a, b}^{l_{x_{a}}}\left(\boldsymbol{x}_{a}\right)-d_{a, b}^{l_{a}}\left(\boldsymbol{y}_{a}\right)\right\|_{1}\right)
\end{array}
$$

The data term involves the matching $\operatorname{cost} C\left(\boldsymbol{x}_{a}, d_{a, b}^{l_{x_{a}}}\right)$ and the inconsistency value $\operatorname{Inc}\left(\boldsymbol{x}_{a}+d_{a, b}^{l_{x_{a}}}\right)$ which is introduced to make it more robust. The regularization term involves motion similarities with neighboring positions. $\alpha_{x_{a}, y_{a}}$ accounts for local color similarities in frame $I_{a}$. Functions $\rho_{d}$ and $\rho_{r}$ 


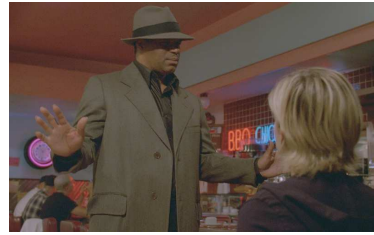

(a) Original image $I_{5036}$

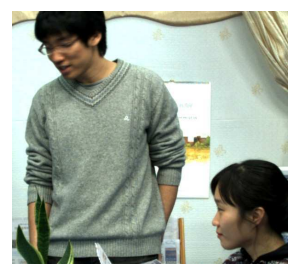

(e) Original image $I_{230}$

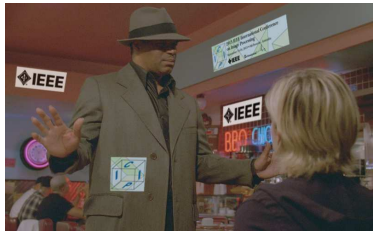

(b) Logo insertion in $I_{5036}$

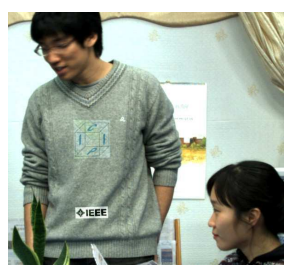

(f) Logo insertion in $I_{230}$

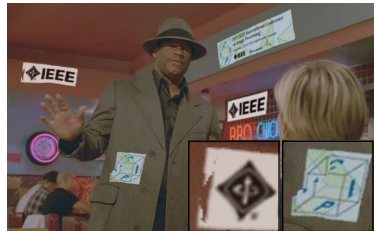

(c) Propagation to $I_{5054}, G O$

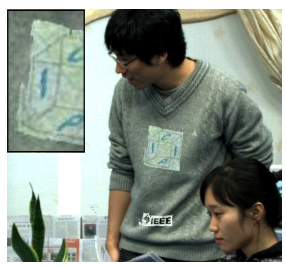

(g) Propagation to $I_{160}, G O$

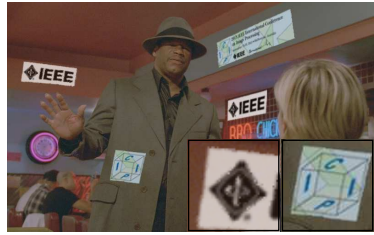

(d) Propagation to $I_{5054}, S P+G O$

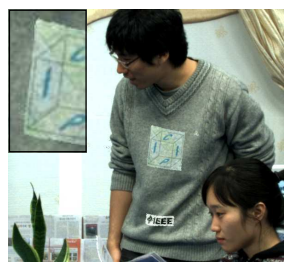

(h) Propagation to $I_{160}, S P+G O$

Fig. 4: a-d) Logo insertion in $I_{5036}$ and propagation to $I_{5054}$ (Hope). e-h) Logo insertion in $I_{230}$ and propagation to $I_{160}$ (Newspaper). We compare the global optimization $(G O)$ method with the statistical processing $(S P)$ combined to $G O(S P+G O)$.

\begin{tabular}{|l|c|c|c|c|c|}
\hline Frame pairs & $\{160,190\}$ & $\{160,200\}$ & $\{160,210\}$ & $\{160,220\}$ & $\{160,230\}$ \\
\hline$G O$ & 21.11 & 19.33 & 18.11 & 17.06 & 16.29 \\
\hline SP+GO & $\mathbf{2 1 . 4 2}$ & $\mathbf{1 9 . 5 3}$ & $\mathbf{1 8 . 3}$ & $\mathbf{1 7 . 7 4}$ & $\mathbf{1 7 . 0 9}$ \\
\hline$M S F[12]$ & 20.5 & 18.22 & 17.8 & 16.95 & 16.6 \\
\hline
\end{tabular}

Table 2: Registration and PSNR assessment with: the combinatorial integration followed by the global optimization $(G O)$; by the statistical processing combined to $G O(S P+G O)$; the multi-step fusion $(M S F)$ method [12]. PSNR scores are computed on the whole images of Newspaper (Fig. 4).

are described in [10]. Fusion moves algorithm fuses candidates pair by pair up to getting an optimal field $d_{a, b}^{*}$ but its application to a large set is limited by the computational load.

\subsection{Motion vector selection framework}

We propose to combine statistical processing and the above global optimization stage to combine simultaneously information about the point distribution, a robust selection based on the intrinsic motion field quality and a spatial regularization. For each $\boldsymbol{x}_{a} \in I_{a}$, the statistical processing is applied to the whole set $T_{a, b}\left(\boldsymbol{x}_{a}\right)$. Then, we select the $N_{o p t}$ best candidates of the distribution with the criterion of median minimization of (3). Finally, fusion moves algorithm fuses by pairs these $N_{\text {opt }}$ candidates up to obtaining the best one.

\section{RESULTS}

Our experiments focus on frame pairs taken from three sequences: MPI S1 [14], Hope and Newspaper. For the selected pairs, the combinatorial multi-step integration has been performed taking input elementary flow fields estimated with a $2 \mathrm{D}$ version of the disparity estimator of [17]. For all the experiments, the parameters are : $N_{c}=7, N_{s}=100, Q_{\max }=$ $2, N_{\text {opt }}=3$. Steps 1, 2, 3, 4, 5, 15 and 30 have been used.

After this motion field construction stage, we have compared three selection procedures: 1) the statistical processing $(S P), 2)$ the global optimization $(G O)$ method solved by $f u$ - sion moves [15], 3) the statistical processing combined with the global optimization $(S P+G O)$. Firstly, the final fields of each method have been compared through registration and PSNR assessment. For a given pair $\left\{I_{a}, I_{b}\right\}$, the final fields are used to reconstruct $I_{a}$ from $I_{b}$ through motion compensation and color PSNR scores are computed between $I_{a}$ and the registred frame for non-occluded pixels. Tables 1 and 2 show quantitative comparisons through PSNR computed for various distances between $I_{a}$ and $I_{b}$ respectively on the kiosk of MPI S1 and on whole images of Newspaper. An example of registration of the kiosk for a distance of 21 frames is provided Fig. 3. Results show that $S P$ is better than $G O$ for all pairs. The low diversity of candidates at the output of $S P$ limits the effect of regularization and explains the slight improvement between $S P$ and $S P+G O$. The example of Fig. 3 is interesting due to the temporary occlusion of the kiosk which is jumped by multi-step motion paths. For this complex situation, $S P+G O$ is more adapted than $G O$. Secondly, in the context of video editing, we evaluate the accuracy of $S P+G O$ and $G O$ by motion compensating in $I_{b}$ logos manually inserted in $I_{a}$. Fig. 4 presents results for Hope and Newspaper with a distance of 18 and 70 frames respectively. For both cases, $S P+G O$ shows a clear improvement compared to $G O$.

The proposed combinatorial integration combined to $S P+G O$ gives better performance compared to the multistep fusion (MSF) method [12] according to PSNR scores of Table 2. The $M S F$ method itself has been shown in [12] to outperform state-of-the-art methods such as $[3,6,7]$.

\section{CONCLUSION}

We perform long-term dense matching by considering multiple multi-step motion paths along the sequence. Given the resulting large set of motion candidates, we apply a selection procedure where the global optimization stage is preceded by a new statistical processing which exploits the spatial distribution and the intrinsic quality of candidates. It leads to better results compared to state-of-the-art methods. 


\section{REFERENCES}

[1] B.D. Lucas and T. Kanade, "An iterative image registration technique with an application to stereo vision," International joint conference on artificial intelligence, vol. 2, pp. 674-679, 1981.

[2] B.K.P. Horn and B.G. Schunck, "Determining optical flow," Artificial Intelligence, vol. 17, no. 1, pp. 185203, 1981.

[3] C. Zach, T. Pock, and H. Bischof, "A duality based approach for realtime TV-L1 optical flow," Pattern Recognition, pp. 214-223, 2007.

[4] Frank Steinbrucker, Thomas Pock, and Daniel Cremers, "Large displacement optical flow computation without warping," in IEEE International Conference on Computer Vision, 2009, pp. 1609-1614.

[5] D. Sun, S. Roth, and M.J. Black, "Secrets of optical flow estimation and their principles," IEEE International Conference on Computer Vision and Pattern Recognition, pp. 2432-2439, 2010.

[6] N. Sundaram, T. Brox, and K. Keutzer, "Dense point trajectories by GPU-accelerated large displacement optical flow," European Conference on Computer Vision, pp. 438-451, 2010.

[7] T. Brox and J. Malik, "Large displacement optical flow: descriptor matching in variational motion estimation," IEEE Transactions on Pattern Analysis and Machine Intelligence, vol. 33, no. 3, pp. 500-513, 2011.

[8] Li Xu, Jiaya Jia, and Yasuyuki Matsushita, "Motion detail preserving optical flow estimation," IEEE Transactions on Pattern Analysis and Machine Intelligence, vol. 34, no. 9, pp. 1744-1757, 2012.

[9] M. W. Tao, J. Bai, P. Kohli, and S. Paris, "SimpleFlow: A non-iterative, sublinear optical flow algorithm," Computer Graphics Forum (Eurographics 2012), vol. 31, no. 2, 2012.

[10] V. Lempitsky, S. Roth, and C. Rother, "FusionFlow: Discrete-continuous optimization for optical flow estimation," IEEE International Conference on Computer Vision and Pattern Recognition, pp. 1-8, 2008.

[11] T. Crivelli, P.-H. Conze, P. Robert, and P. Pérez, "From optical flow to dense long term correspondences," in IEEE International Conference on Image Processing, 2012.

[12] T. Crivelli, P.-H. Conze, P. Robert, M. Fradet, and P. Pérez, "Multi-step flow fusion: Towards accurate and dense correspondences in long video shots," in British Machine Vision Conference, 2012.
[13] J. Wills and S. Belongie, "A feature-based approach for determining dense long range correspondences," $E u$ ropean Conference on Computer Vision, pp. 170-182, 2004.

[14] M. Granados, K. I. Kim, J. Tompkin, J. Kautz, and C. Theobalt, "MPI-S1," http://www.mpi-inf . mpg.de/ granados/projects/vidbginp/ index.html.

[15] V. Lempitsky, C. Rother, S. Roth, and A. Blake, "Fusion moves for Markov random field optimization," IEEE Transactions on Pattern Analysis and Machine Intelligence, vol. 32, no. 8, pp. 1392-1405, 2010.

[16] L. Yin, R. Yang, M. Gabbouj, and Y. Neuvo, "Weighted median filters: a tutorial," Circuits and Systems II: Ana$\log$ and Digital Signal Processing, IEEE Transactions on, vol. 43, no. 3, pp. 157-192, 1996.

[17] P. Robert, C. Thébault, V. Drazic, and P.-H. Conze, "Disparity-compensated view synthesis for s3d content correction," in SPIE IS\&T Electronic Imaging Stereoscopic Displays and Applications, 2012. 